\title{
Completing the Picture: Compositional Data Obtained with Energy Dispersive X- ray Spectrometry Contributes Novel Information for Stained and Unstained Biological Samples
}

\author{
Louise Hughes ${ }^{1}$ and Errin Johnson ${ }^{2}$ \\ ${ }^{1}$ Oxford Instruments NanoAnalysis, High Wycombe, England, United Kingdom, ${ }^{2}$ University of Oxford, \\ Oxford, England, United Kingdom
}

Visualisation and analysis of biological samples has been a challenge throughout the history of electron microscopy with sample preparation and the absence or addition of contrasting agents playing a key role in the development of imaging methodology. Multi-colour electron microscopy (MCEM) combines ultrastructural electron data with elemental information about sample composition [1]. While energy dispersive $\mathrm{x}$-ray spectrometry (EDX) has been used in materials science and biology for many decades, sample stability and detector sensitivity have limited the broader adoption in life sciences until recently $[2]$.

Ongoing questions in biological MCEM include what effect sample preparation has on the elemental mapping of samples and how well EDX of stained, embedded material is applicable to biological research. We used a variety of biological samples, such as macrophages, erythroblasts, heart tissue, rice roots and leaves, that have been stained using a combination of traditional methods or have been prepared without the addition of contrasting agents to compare the reproducibility of elemental mapping in addition to production of analytical data.

Our results showed that endogenous biological elements are preserved during routine sample preparation and even when stains are used it is possible to analyse and map all essential elements and some trace elements in select specimens. A more significant variable in the process appears to be the sample itself, with plant tissue demonstrating a robust preservation of native elements.

Further analysis of our results indicated that there is a greater variability to common staining agents than is recorded in literature. The zinc-iodide-osmium (ZIO) stain, often utilised for staining endomembrane systems [3] and cited as having a 3:2 or 4:1 ratio of osmium to zinc in electron dense precipitates [4], showed a significant differential distribution and a variety of osmium and zinc ratios in cells and tissues that was not always reflected in electron contrast (fig. 1). Similar results were observed in other common stains.

EDS provided a significant advantage with several specimens in providing identifying ultrastructural evidence that was absent in electron data. For instance, the addition of elemental maps to electron images contributes key information that could be used for a variety of biological imaging applications, such as region of interest profiling or automated segmentation of volumetric data (fig. 2). EDS also introduces the possibility of employing different staining agents or labels to specimens. 

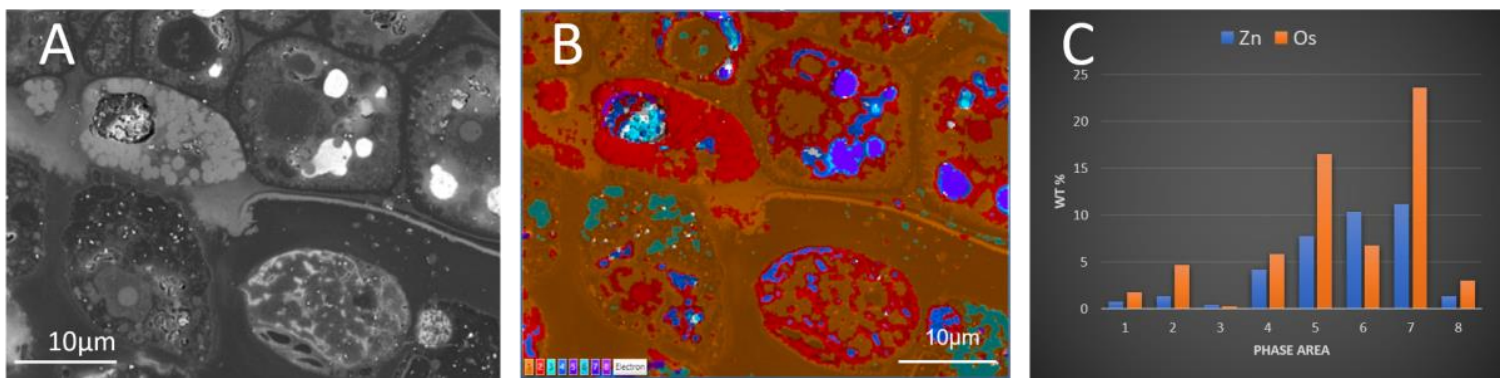

Figure 1. Backscattered electron image of a digestive gland on a Venus' fly trap leaf (A), phase map based on EDS analysis of the sample element distribution (B) and zinc and osmium wt\% values (C) for the phases shown in the phase map image.
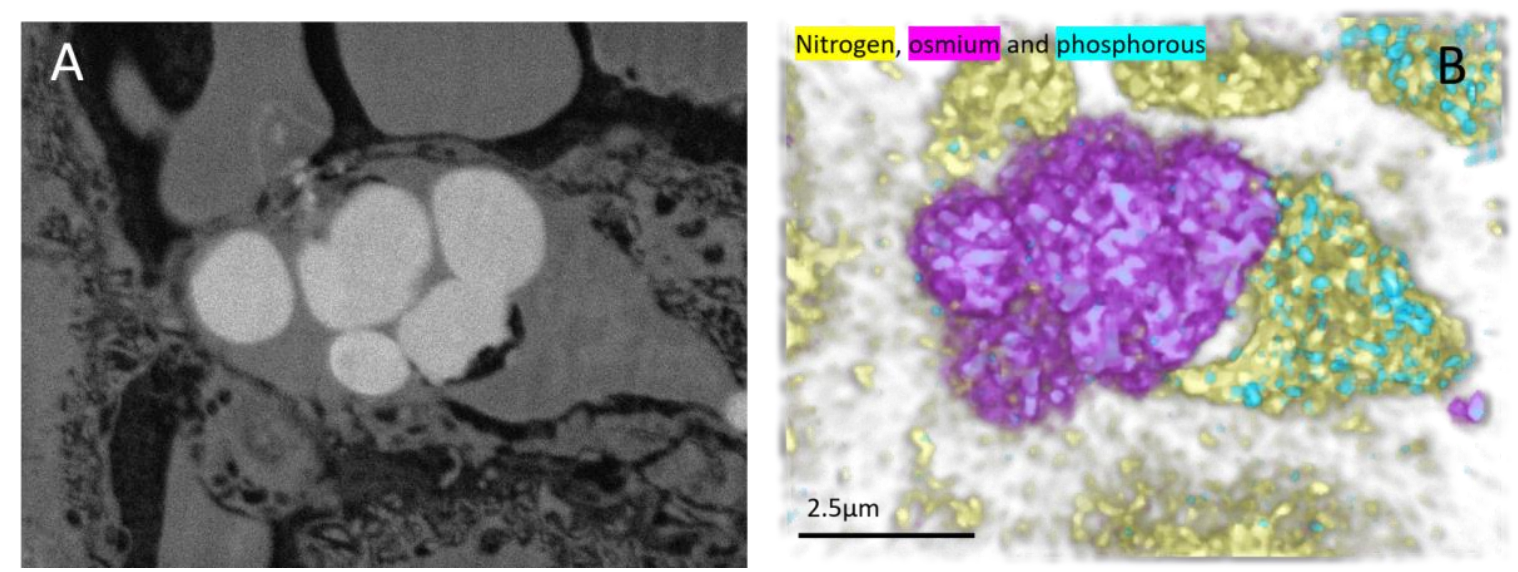

Figure 2. Backscattered electron image of liver tissue (A) and the 3D reconstruction of EDS maps showing the distribution of nitrogen (yellow), osmium (pink) and phosphorous (blue).

\section{References}

[1] Scotuzzi, M., Kuipers, J., Wensveen, D.I., De Boer, P., Hoogenboom, J.P. and Giepmans, B.N., 2017. Multi-color electron microscopy by element-guided identification of cells, organelles and molecules. Scientific reports, 7(1), pp.1-8.

[2] Pirozzi, N.M., Hoogenboom, J.P. and Giepmans, B.N., 2018. ColorEM: analytical electron microscopy for element-guided identification and imaging of the building blocks of life. Histochemistry and cell biology, 150(5), pp.509-520.

[3] Kittelmann, M., Hawes, C. and Hughes, L., 2016. Serial block face scanning electron microscopy and the reconstruction of plant cell membrane systems. Journal of microscopy, 263(2), pp.200-211.

[4] M. Hayat (2000), Principles and techniques of electron microscopy, biological applications. Cambridge University Press, Cambridge. 